GEM tracker for high luminosity experiments at the JLab Hall A

This article has been downloaded from IOPscience. Please scroll down to see the full text article.

2012 JINST 7 C05013

(http://iopscience.iop.org/1748-0221/7/05/C05013)

View the table of contents for this issue, or go to the journal homepage for more

Download details:

IP Address: 137.138.124.233

The article was downloaded on 26/07/2012 at $16: 12$

Please note that terms and conditions apply. 
$2^{\text {nd }}$ International Conference on Micro Pattern Gaseous Detectors, 29 August - 1 SEPTEMBER 2011,

KOBE, JAPAN

\title{
GEM tracker for high luminosity experiments at the JLab Hall A
}

\author{
V. Bellini, ${ }^{a}$ E. Cisbani, ${ }^{b, c, 1}$ M. Capogni, ${ }^{b, d}$ S. Colilli, ${ }^{b, c}$ R. De Leo, ${ }^{e}$ R. De Oliveira, ${ }^{f}$ \\ V. De Smet, ${ }^{a, g}$ R. Fratoni, ${ }^{b, c}$ S. Frullani, ${ }^{b, c}$ F. Giuliani, ${ }^{b, c}$ M. Gricia, ${ }^{b, c}$ F. Librizzi, ${ }^{a}$ \\ M. Lucentini, ${ }^{b, c}$ F. Mammoliti, ${ }^{a}$ S. Minutoli, ${ }^{h}$ P. Musico,${ }^{h}$ F. Noto,${ }^{a}$ R. Perrino, ${ }^{i}$ \\ F. Santavenere ${ }^{b, c}$ and C. Sutera ${ }^{a}$ \\ ${ }^{a}$ INFN Catania and Catania University, \\ via Santa Sofia 64, I-95123 Catania, Italy \\ ${ }^{b}$ INFN Roma Sanità Group, \\ viale Regina Elena 299, I-00161 Rome, Italy \\ ${ }^{c}$ Italian National Institute of Health, \\ viale Regina Elena 299, I-00161 Rome, Italy \\ ${ }^{d}$ ENEA Centro Ricerche Casaccia, \\ via Anguillarese 301, I-00123 Santa Maria di Galeria (Rome), Italy \\ ${ }^{e}$ INFN Bari and Bari University, \\ via E. Orabona 4, I-70125 Bari, Italy \\ ${ }^{f}$ European Organization for Nuclear Research CERN, \\ CH-1211 Genève 23, Switzerland \\ ${ }^{g}$ Istitut Supérior Industriel De Bruxelles, \\ rue Royale 150, B-1000 Bruxelles, Belgium \\ ${ }^{h}$ INFN Genova, \\ via Dodecaneso 33, I-16146 Genova, Italy \\ ${ }^{i}$ INFN Lecce, \\ Via Arnesano, I-73100 Lecce, Italy \\ E-mail: evaristo.cisbani@iss.infn.it
}

\footnotetext{
${ }^{1}$ Corresponding author.
} 
ABSTRACT: A new large-area, lightweight tracker based on the GEM technology is under development for the upcoming experiments in Hall A at Jefferson Lab, where a longitudinally polarized electron beam of $11 \mathrm{GeV}$ will be available in late 2013. This beam, combined with innovative polarized targets, will provide luminosity up to $10^{39} /\left(\mathrm{s} \cdot \mathrm{cm}^{2}\right)$ opening exciting opportunities to investigate unexplored aspects of the inner structure of the nucleon and the dynamics of its constituents. The GEM tracker design is presented in this paper.

KEYwords: Micropattern gaseous detectors (MSGC, GEM, THGEM, RETHGEM, MHSP, MICROPIC, MICROMEGAS, InGrid, etc); Particle tracking detectors (Gaseous detectors); Front-end electronics for detector readout 


\section{Contents}

1 Introduction 1

2 GEM Tracker 2

2.1 GEM $40 \times 50 \mathrm{~cm}^{2}$ basic module 3

3 Readout electronics $\quad 6$

4 Design analysis 6

5 GEANT4 simulation and GEM digitization $\quad 7$

6 Prototype test 9

$\begin{array}{lll}7 & \text { Conclusions } & 11\end{array}$

\section{Introduction}

The Jefferson Laboratory (JLab) [1] is one of the most important experimental facility providing a multi $\mathrm{GeV}$, high intensity, longitudinally polarized, electron beam; the origin of the quark and gluon confinement, the dynamics of the quarks and gluons in the nucleon and of the nucleon in the nucleus, the structure of the nuclei and the limits of the standard model are some of the most relevant fields of fundamental physics investigations at JLab.

The laboratory is undergoing a major upgrade of its Continuous Electron Beam Accelerator Facility (CEBAF) and experimental halls. In late 2013, the accelerator will deliver electrons with energy up $12 \mathrm{GeV}$ (twice the present limit) with excellent intensity (up to $100 \mu \mathrm{A}$ ) and longitudinal polarization (up to $85 \%$ ). The equipments of the three existing experimental halls are under upgrade to optimally exploit the opportunities of the new beam.

Hall A, the largest experimental hall, presently equipped with two high resolution (HRS) and a moderate acceptance (BigBite) spectrometers and additional dedicated detectors, will be extensively devoted to high luminosity experiments (thanks also to the developments of new fixed targets). In this direction, activity is ongoing to implement the new Super BigBite Spectrometer (SBS) [2] with large acceptance (both in angle and momentum), capability to operate in very high luminosity environment (up to $10^{39} /\left(\mathrm{s} \cdot \mathrm{cm}^{2}\right)$ ) and at forward scattering angle (down to 7 degree), easily reconfigurable to best fit the experimental needs. The new spectrometer will consist (refer to figure 1) of a dipole magnet with field integral up to $3 \mathrm{~T} \cdot \mathrm{m}$ (it will operate at about $2 \mathrm{~T} \cdot \mathrm{m}$ ), a primary charged particle tracker (front tracker), two identical proton polarimeters (made of a Carbon analyzer and large tracker), a hadron calorimeter; the proton polarimeters can be replaced by one of the two modules of the HERMES RICH ${ }^{1}$ [3] sitting at 90 degree respect to the original set up in the HERMES experiment.

\footnotetext{
${ }^{1}$ One of the HERMES RICH modules, with spare parts, is currently safely stored at the University of Virginia.
} 


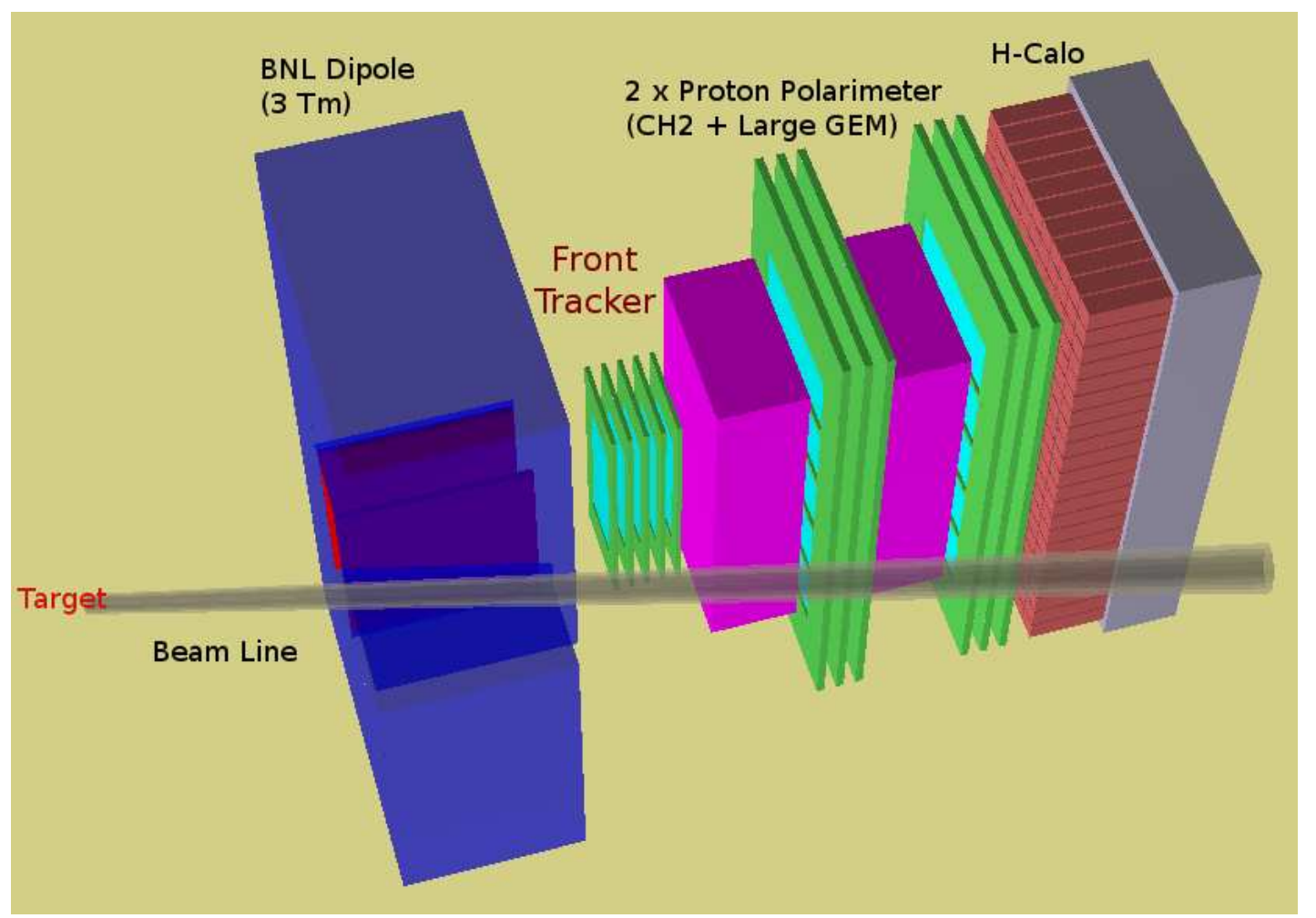

Figure 1. Preliminary schematic layout of the SBS spectrometer; the front tracker is represented by 5 GEM detectors out of 6 expected in the complete setup.

SBS will initially serve four experiments [4] dedicated to the study of the nucleon structure in terms of elastic electromagnetic form factors at high 4-momentum transfer $Q^{2}$ up to $15 \mathrm{GeV}^{2}$ and of transverse momentum distributions of the quarks in the Semi Inclusive Deep Inelastic (SIDIS) region.

The tracking systems of SBS will be mainly based on triple-GEM detectors with analog readout as detailed later. In the next sections the front tracker of SBS will be described, along with the most relevant features. One section will be dedicated to the readout electronics based on the APV25 [5] chip. Some details of the design analysis will be shortly summarized. The preliminary test beam results will be discussed in the last section.

\section{GEM Tracker}

The main requirements of the SBS front tracker are:

1. hit spatial resolution at the level of $70 \mu \mathrm{m}$,

2. capability to stand large background flux $\left(250 \mathrm{MHz} / \mathrm{cm}^{2}\right.$ photons and $160 \mathrm{kHz} / \mathrm{cm}^{2}$ charged particles, mainly electrons),

3. coverage of a transverse area of about $40 \times 120 \mathrm{~cm}^{2}$,

4. reconfigurable (reuse of the same detector in different configurations),

5. event acquisition rate at the level of $20 \mathrm{k}$ event/s. 


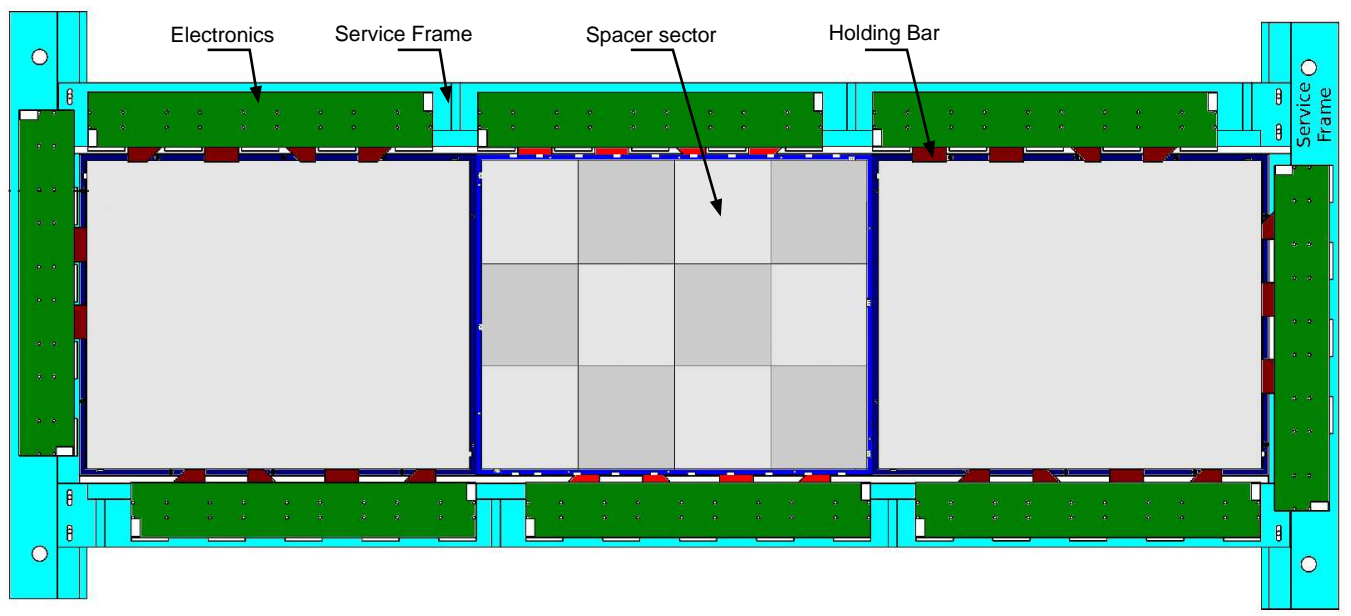

Figure 2. Layout of a single GEM chamber, composed of three $40 \times 50 \mathrm{~cm}^{2}$ GEM modules; spacer sectors are represented in the middle module.

The first and second requirements, taking into account the moderately large area (and therefore costs), are well fulfilled by the recent GEM technology; in particular the high particle flux which corresponds to a hits rate on the tracker entrance at the level of $500 \mathrm{kHz} / \mathrm{cm}^{2}$ (from simulation, the reduction is due to the low efficiency of the gamma photon detection) is well below the stable gain limit $\geq 10 \mathrm{MHz} / \mathrm{cm}^{2}$ reported in literature [6]. In fact, the front tracker is based on 6 high resolution, large GEM detectors (chambers), for a total tracker length of about $50 \mathrm{~cm}$. Each chamber, schematically presented in figure 2 has an active area of $40 \times 150 \mathrm{~cm}^{2}$, made by 3 adjacent GEM modules; each module has $40 \times 50 \mathrm{~cm}^{2}$ active rectangular area, for a total of 18 modules.

The modules are largely independent, characterized by their own readout, high voltage supply and gas inlet/outlet as well as front-end electronics; these features provide adequate modularity to fulfill the aforementioned requirement for a reconfigurable detector.

\subsection{GEM $40 \times 50 \mathbf{c m}^{2}$ basic module}

The size of the GEM $40 \times 50 \mathrm{~cm}^{2}$ module has been defined considering the following aspects:

- technology limits on GEM foil production: at the time the project began, the double mask technique was the only consolidated way for the construction of good quality GEM foils; the precise alignment required by this technique limits the linear size of the foil to less than $60 \mathrm{~cm}$.

- noise and pile-up: longer strip are generally more noisy due to the increased strip capacitance and higher probability of noise pick up from the environment. Moreover, longer strip has higher hits pile up, which degrades the track reconstruction capability.

- experimental requirements in terms of tracker reconfiguration: the GEM tracker will be used, in different geometries both in the SBS and in BigBite spectrometers (in particular for the SIDIS experiment); moreover, the same module will be the base component of chambers of different size and characteristics used as polarimeter tracker in SBS or single coordinate detector in front of a segmented calorimeter. 

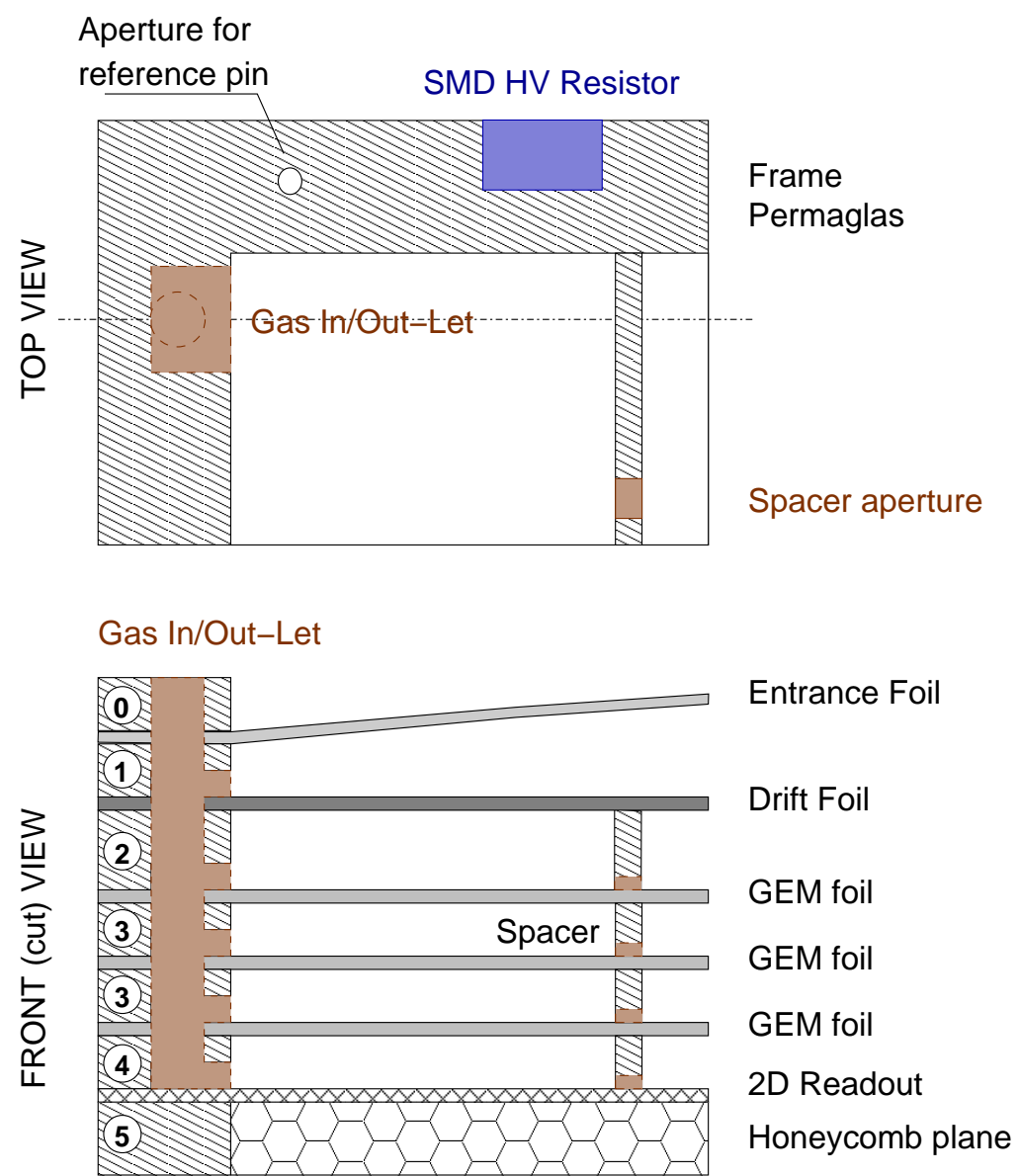

Figure 3. Schematic top and side views of part of the GEM module. All relevant elements are represented. The numbers distinguish the different frames of the module.

The main mechanical support of the single GEM module (refer to figure 3 ) is one $3 \mathrm{~mm}$ thick honeycomb plane where the readout foil is directly glued. The readout foil is made of two strip layers (same design of COMPASS [7]) that represent the $\mathrm{x}$ and $\mathrm{y}$ coordinates; strip pitch is $0.40 \mathrm{~mm}$ in both layer; the lower layer is made of $0.340 \times 400 \mathrm{~mm}^{2}$ strips while the upper layer has strips of $0.080 \times 500 \mathrm{~mm}^{2}$. Three identical cascaded GEM foils are glued on $2 \mathrm{~mm}$ thick frames; the first GEM sits at $2 \mathrm{~mm}$ above the readout. A metalized foil provides the drift of the GEM detector, with a drift gap of $3 \mathrm{~mm}$; a mylar foil on top of the drift foil guarantees the gas tightness and avoids the drift deformation due to the gas pressure.

All frames between the drift foil and the readout foil include internal thin spacers (300 $\mu \mathrm{m}$ width) which help keeping the flatness of the foils, as mentioned later. The spacers have small apertures to facilitate the gas flow. The grid of spacers form the spacer sectors represented in the middle module in figure 2 and visible in the prototype picture reported in figure 7.

The charge collected on the strips is transferred to the electronics through FPC (Flexible printed Circuit) with ZIF (Zero Insertion Force) terminals that avoid soldering of connectors on the readout foil; ${ }^{2}$ the detailed layout of GEM and readout foils (superimposed) is shown in figure 4.

\footnotetext{
${ }^{2}$ The connectors are only on the front-end electronics; in the specific application the 73FXZ-RSM1-6-ETF(LF)(SN) connectors by JST [9] are used.
} 


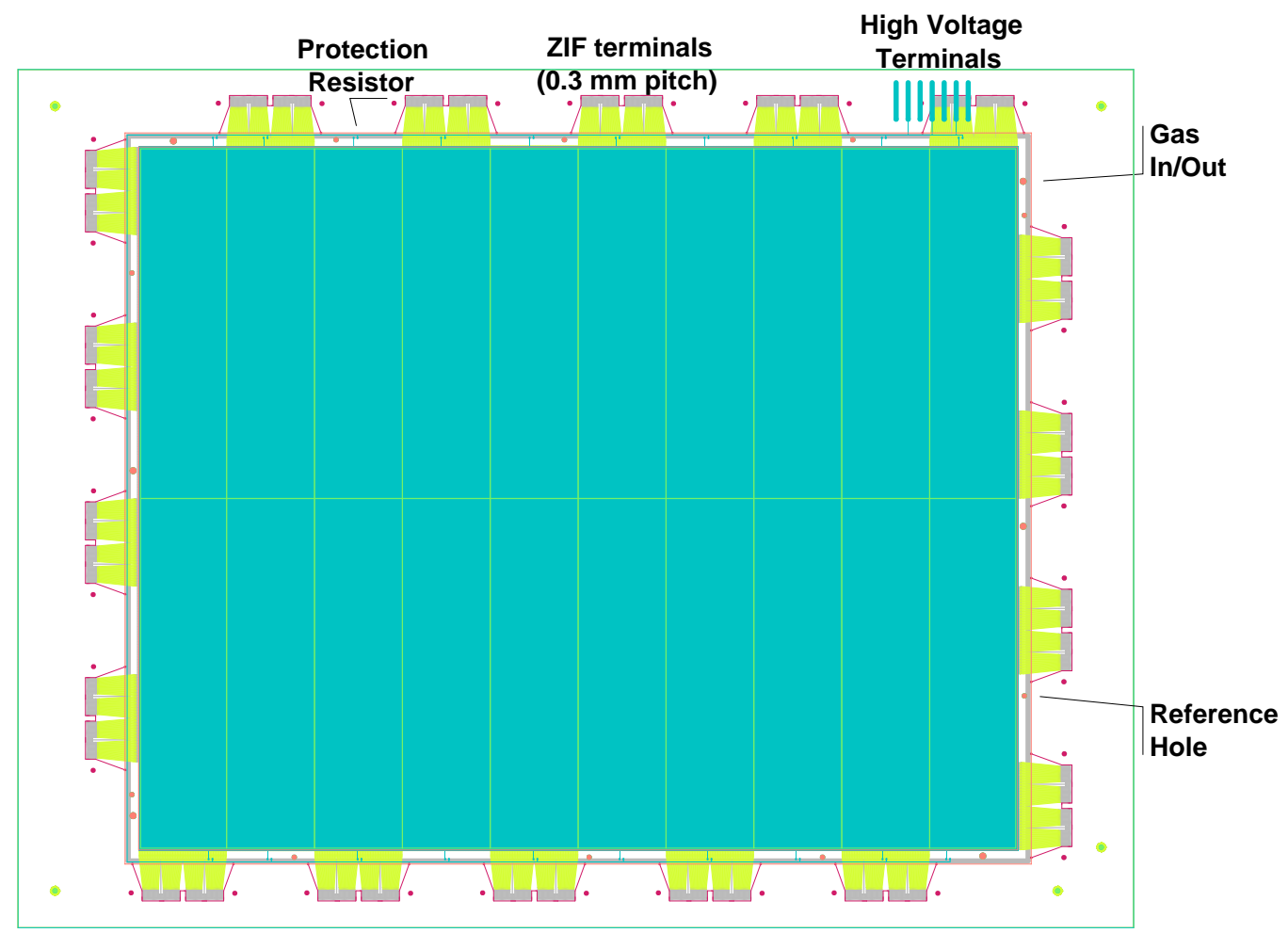

Figure 4. Layout of GEM foil and readout foil; clearly visible the $20 \mathrm{HV}$ sectors, the 7 high voltage contacts near the top-right edge and the 18 readout ZIF terminals distributed along the perimeter. Four HV contacts are connected to the bottom copper layer, three to the $20 \mathrm{HV}$ sectors; only two contacts are used to power a single foil. Each ZIF terminal connects 128 strips to one front-end card.

One side of the GEM foils is divided in twenty $5 \times 20 \mathrm{~cm}^{2}$ sectors to avoid discharge propagation; each sector has its own SMD (Surface Mount Device) protection resistor, placed inside the $2 \mathrm{~mm}$ thin, $8 \mathrm{~mm}$ wide composite laminate frame (PERMAGLAS ME 730 [8]) where the foils are glued.

Drift, GEM and readout foils are fabricated by the CERN TD-DEM Photo Mechanical Technologies group.

As mentioned above, the size of the GEM foil was driven by the double mask technique; however, considering the achieved production quality of the single mask, the current performance (comparable to the double mask, in terms of gain and leakage current) and the lower cost, the single mask will be adopted in the final production.

The modules are integrated in a way to minimize the dead area and are supported by an outer carbon fiber frame (the service frame shown in figure 2) to keep thermal deformation small enough to achieve the required spatial resolution; this frame is also used to support all services: readout electronics, signal cabling, gas pipes, HV components.

The module internal volume is expected to be continuously flushed by a gas mixture of Ar $(70 \%)$ and $\mathrm{CO}_{2}(30 \%)$. 


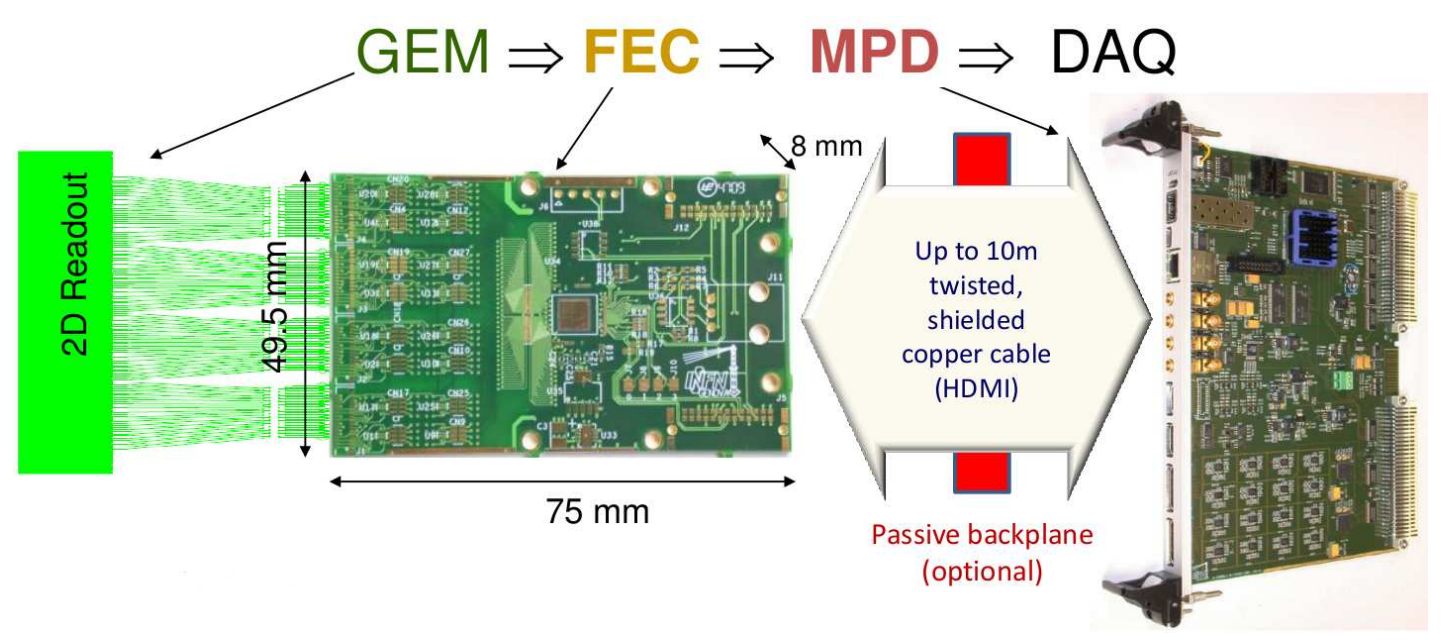

Figure 5. The readout electronics chain based on the APV25 chip, developed for the GEM tracker.

\section{Readout electronics}

Compactness, simplicity and versatility have driven the design of the readout electronics based on the 128 channel APV25 ASIC [5]. Each APV25 channel consists of a preamplifier followed by a shaper and a 192 cell analog pipeline, where the shaped signal is continuously sampled at 20 or $40 \mathrm{MHz}$ (user selectable). The samples awaiting readout are flagged by external triggers, with adjustable latency.

As shown in figure 5, the readout electronics consists of two active components: the frontend cards, directly connected to the detector strips and hosting the APV25, and a multi-purpose digitizer board (MPD).

One MPD handles up to 16 front-end cards (for a total of 2048 channels) and can be used in VME environments (also VME64x or VXS). It is managed by an ALTERA Arria GX FPGA and it includes sixteen 12 bits A/D converters synchronously running at $40 \mathrm{MHz}$, and ancillary electronics for driving the I2C protocol used to configure the APV25, as well as Ethernet 10-100, and SFP (Small Form-Factor Pluggable) Optical transceiver, for system diagnostics and future extension; $2 \times 64$ Mbyte SDRAM is used to buffer the data and 8 front panel coaxial I/O connectors are provided to interface external control signals.

The FPGA performs several real-time tasks that maximize the data transfer rate: common noise and pedestal subtraction, sparse readout, trigger-uncorrelated hits suppression (based on the multi sample capability of the APV25), event building.

The electronics system is flexible enough to be used in other applications and experiments with minimal modification; in fact its architectural simplicity, use of standard components and scalability, make it suitable to small scale experiments, such as the Olympus experiment at DESY [10], where the very same electronics is used to readout six $10 \times 10 \mathrm{~cm}^{2}$ GEM chambers with 2 dimensional readout (pad-strip configuration, $0.40 \mathrm{~mm}$ pitch).

\section{Design analysis}

Mechanical structure and gas flow have been optimized by analytical considerations and finite element analysis (in the COMSOL framework [11]). 
The GEM foil will be stretched before gluing to guarantee adequate flatness of the GEM and drift foils (deformation less than $1 \%$ of the gap width between foils). In fact, the maximum foil deformation $u$ depends on the foil linear tension $T$, on the pressure $P$ due to the electrostatic fields, and the largest unconstrained surface $S$ of the foil (the area of the spacer sectors mentioned above and represented in figure 2); as a first approximation, the above quantities are related by [12]:

$$
u=k \cdot \frac{P \cdot S}{T}
$$

where $k$ is a function of the geometry of the surface $S$ (for rectangular shape, the maximum value of $k$ is about 0.7367 which corresponds to a square).

Assuming the foil is one of the two parallel planes of a capacitor with $2 \mathrm{~mm}$ gap, with a field of $5 \mathrm{kV} / \mathrm{cm}$, the electrostatic pressure on the foil is at the level of $0.1 \mathrm{~Pa}$, which may become larger during a discharge; to account this fact, a safe factor of 100 is applied to the above electrostatic pressure $^{3}$ to get $P=10 \mathrm{~Pa}$. With a target deformation of $u \leq 20 \mu \mathrm{m}$, the above expression becomes $S \leq 266 \cdot T$ where $S$ is expressed in $\mathrm{cm}^{2}$ and $T$ in $\mathrm{kg} / \mathrm{cm}$. Taking into account that the maximum tension that does not deform noticeably the honeycomb structure is at the level of $T \sim 1 \mathrm{~kg} / \mathrm{cm}$, the surface of the sector spacer must be $S \leq 266 \mathrm{~cm}^{2}$. This approximate analytical result has been refined by a finite element analysis showing that with a spacer sector of $170 \mathrm{~cm}^{2}$ a maximum sag of the foil below $10 \mu \mathrm{m}$ is expected.

The gas flow has been analyzed by means of a 2D model: number of in/out-lets, apertures in the frame spacers and positions have been chosen in order to maximize homogeneity of the flow [13]. All inlets and outlets have the same shape that has been modeled in the attempt to minimize the gas turbulence; in fact, these openings represent the most critical point for the laminar flow of the gas. The final design is presented in figure 6; the choice of the inlet and outlet positions has taken into account the gas flow, the rigidity of the frame and the better accessibility of the edges of the modules, once composed into a chamber.

\section{GEANT4 simulation and GEM digitization}

Tracking performance of the system has been investigated by Monte Carlo events. The GEANT4 simulation framework [14] has been used to model the SBS spectrometer and the GEM tracker in detail. ROOT [15] tree output has been used to store the simulated hits and the digitized data.

The digitization algorithm implements the basic physics processes that generate the electronics signals in the readout strips and the corresponding pulses coming out from the readout electronics from the deposited energy $\Delta E$ of the primary particle in each GEM chamber of the tracker, simulated by the GEANT4 code.

The main processes modeled by the digitization are:

- Ionization: the number of ion pairs is distributed according to a Poissonian distribution with mean parameter $n_{i o n}=\Delta E / W_{i}$; the ionization point is assumed to be uniformly distributed along the primary particle track in the drift region of the GEM;

\footnotetext{
${ }^{3}$ The large safe factor tries to take into account the considerable flux of ionizing particles expected in the high luminosity experiments where the GEM chambers will operate; for the same reason the maximum deformation of the foil has been chosen very restrictively $(\sim 1 \%)$, as long as it does not impact significantly on the design of the mechanical support.
} 


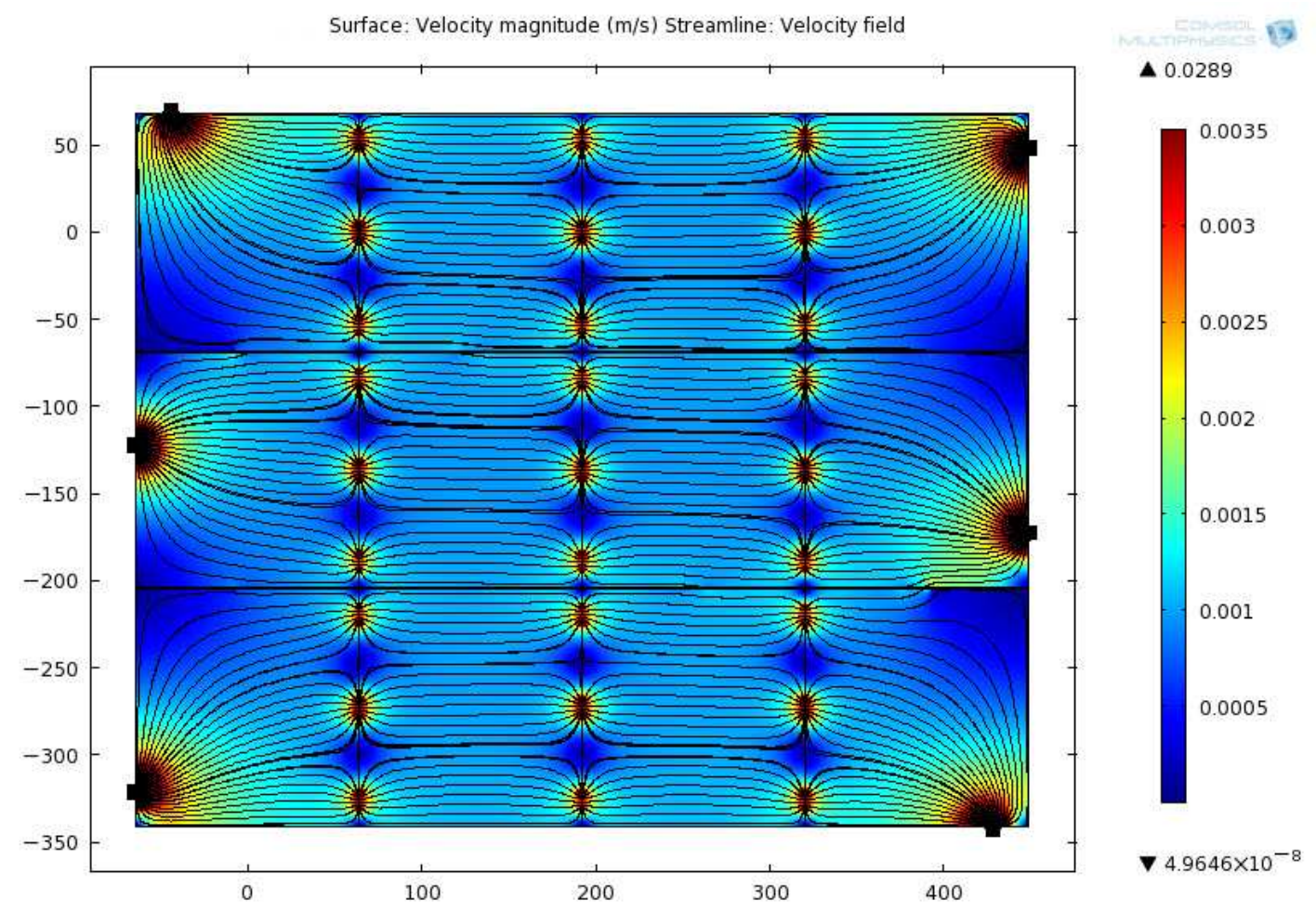

Figure 6. Gas flow analysis; final design. The colors represent the velocity field magnitude in $\mathrm{m} / \mathrm{s}$ (scale on the right); the black lines represent the gas velocity field. Critical regions, in terms of turbulence, are the 3 inlets and 3 outlets and to a lesser extend the apertures of the vertical spacers. Flow is basically horizontal (in the picture): there is no significant mixtures between different vertical sectors (there are four barely visible apertures in each horizontal spacer).

- Drift: the average drift time is given by $t_{d r i f t} \sim L / v_{d}$, where $L$ is the distance between the center of the drift region of the GEM and the readout plane, while $v_{d}$ is the drift velocity (typically $v_{d}=5 \div 6 \mathrm{~cm} / \mu \mathrm{s}$ );

- Diffusion: the spatial distribution of the charge in the readout plane follows some sort of distribution (from gaussian to rectangular) with standard deviation $\sigma_{s}=\sqrt{2 D L / v_{d}}$, where $D$ is the diffusion coefficient $\left(D=200 \div 300 \mathrm{~cm}^{2} / \mathrm{s}\right)$;

- GEM multiplication: the distribution of the number of secondary particles is assumed to follow either the Poissonian or the Furry distributions, the limits of the Polya distribution [16];

- Charge collection: the spatial distribution of the collected charge is assumed to follow the 2D Heaviside step function, which reproduces reasonably well the existing data [7], with spatial width defined by the $\sigma_{s}$ provided above;

- Pulse formation: the shape of the analog pulse coming out from the electronics (APV25) is represented by a double exponential [17].

The digitization results are tuned by means of the GEM COMPASS data [7]. 


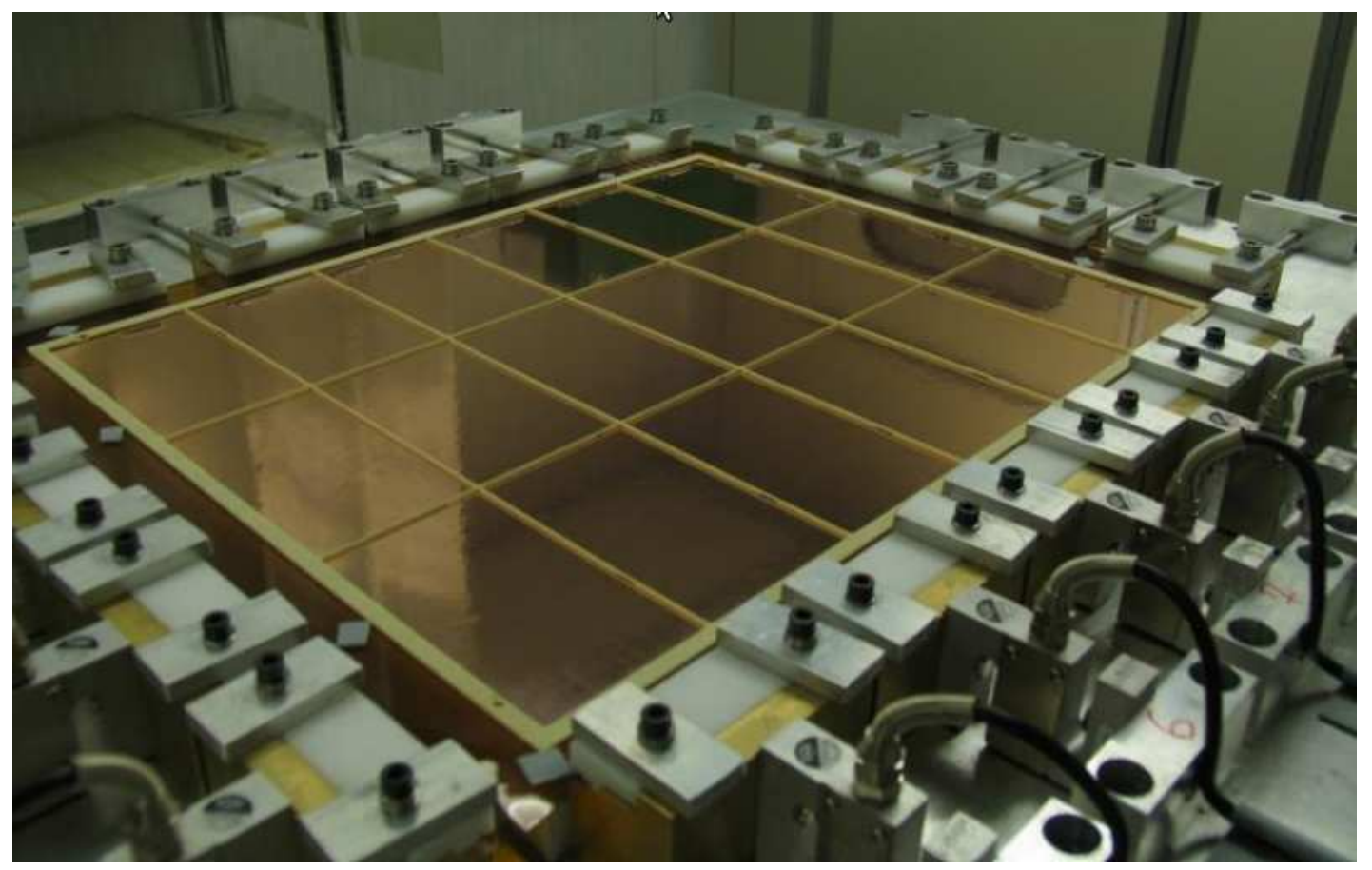

Figure 7. First full scale GEM module prototype during assembling. Three components are visible: the stretched GEM foil; the thin support PERMAGLAS frame with the spacers which form 18 open sectors (their configuration is different respect to the final version presented in figure 2 and 6); the foil stretcher, whose design is derived from [20].

Hits from signal and background particles can be mixed after the digitization process; the number of background hits $n_{b c k}$ has been assumed to follow a Poisson distribution with mean parameter $r_{b c k} \cdot \Delta t$, where $r_{b c k}$ is the background rate and $\Delta t$ is the effective trigger time window (depending on the width of the shape signal coming out from the readout electronics).

The simulated and digitized data have been reconstructed by the track reconstruction algorithm used in HallA at JLab, adapted and improved for the GEM tracker [18]. The main outcome of the analysis shows that the tracking efficiency is at the level of $90 \%$ at full expected background; the $10 \%$ tracking inefficiency comes from the background hits that cannot be disentangled from the signal and therefore they cause a degradation of the track resolution which do not pass the reconstruction quality cuts (when half of the expected background is simulated the tracking efficiency becomes larger than $96 \%$ ).

Large part of the background hits is removed by time correlation to the trigger, exploiting three consecutive samples of the APV25 shaped signal. The time correlation is also used to suppress hits ambiguity; in fact, the analysis has also shown that chambers with $\mathrm{u} / \mathrm{v}$ strips in addition to the $\mathrm{x} / \mathrm{y}$ ones do not improve noticeably the ambiguity suppression.

\section{Prototype test}

The first prototype of the full scale module with the newly developed readout electronics has been tested at the DESY-II 1 to $6 \mathrm{GeV}$ lepton beam at the end of 2010; test has been partially supported by the EUDET program [19]. 


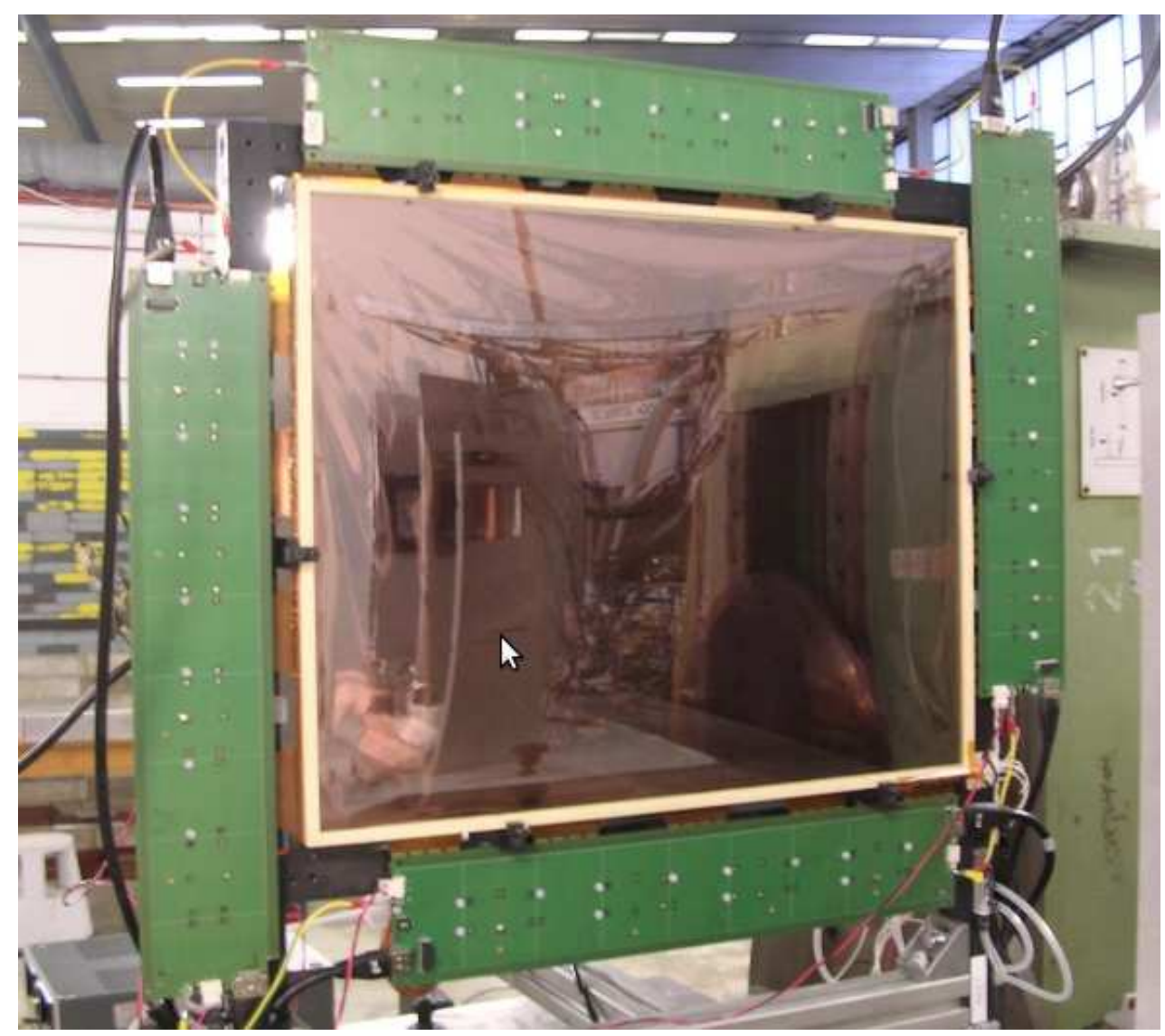

Figure 8. First GEM module prototype, from the drift side (protective Mylar foil visible). The 18 front-end cards are located behind the 4 rectangular backplanes that sit along the 4 sides of the module.

Picture in figure 8 shows the test setup: the module is equipped with the APV25 electronics; precise tracking has performed by small silicon strip detectors located before the GEM; $\operatorname{Ar}(70 \%)$ and $\mathrm{CO}_{2}(30 \%)$ gas mixture has been used. The GEM HV has been powered by the first version of the HV-GEM system ${ }^{4}$ providing 7 independent HV levels.

Two typical events collected at the test are shown on figure 9: on the upper 2 plots, a good event with a single hit, at the center of the module, with large charge, well above the noise level. On the lower 2 plots a noisy event effected by apparently random misalignment of the raw acquired data due to bugs in the electronics firmware, which have been lately fixed. This misalignment causes a wrong compensation of the pedestal, ending up with different types of periodic pattern which obfuscates the signal. These bugs make a comprehensive analysis of the data quite difficult.

Different criteria have been studied to select good events; the most stable criterion consists of the following steps:

1. each axis is divided in two regions: beam region (where the beam is expected to pass), empty region (where no signal is expected); the beam region is about $2 \mathrm{~cm}$ wide, while the empty region is $36 \mathrm{~cm}$ or $46 \mathrm{~cm}$ wide, depending on the axis.

\footnotetext{
${ }^{4}$ The HV-GEM is under development at the Laboratori Nazionali di Frascati [21].
} 
2. the root mean square of the strip charge $\sigma$ is evaluated in the empty region; it represents the level of noise;

3. the charge maximum $s$ and its position $p_{s}$ are searched in the beam region;

4. a narrow symmetric peak region is defined around $p_{s}, 6 \mathrm{~mm}$ wide;

5. the mean charge $b$ is computed in the beam region (excluding the peak region); $b$ represents the baseline of the signal;

6. the event passes the selection if: $(s-b)>2 \sigma$ for both axes.

Once the good events have been selected, adjacent hits are grouped in clusters and the $\mathrm{x}$ and y cluster charges ${ }^{5}$ are estimated. The $\mathrm{x}-\mathrm{y}$ charge correlation goes up to about $90 \%$ (figure 10), and signal and noise distributions look rather good (figure 11) although they are still biased by the quality cuts. These results are preliminary, but, give indications of the general validity of the hardware design and of some critical aspects that have been investigated in the past months.

Recent test in high intensity gamma and electron background has been carried on at MAMI (Mainzer Mikrotron) at the beginning of September 2011, showing excellent on-line data without noisy events like the one presented above. Analysis is in progress.

Since the GEM tracker will seat behind a momentum analyzing spectrometer, where the expected magnet fringe field on the first chamber is at the level of 100 Gauss, oriented prevalently perpendicular to the GEM electrostatic field, new tests are foreseen in the first quarter of 2012 to measure the performance in a low intensity uniform magnetic field (up to 500 Gauss).

\section{Conclusions}

Most of the design of the GEM based tracker and its readout electronics is finalized; analytical considerations and numerical simulations have been used to verify and optimize the adopted solutions. The assembling of the first full scale prototype, late in 2010, has been very useful to improve details of the design and of the ancillary tools, such as the GEM stretcher. First beam test on the prototype has been performed at the end of 2010; some bugs of the preliminary electronics firmware have affected the quality of the acquired data. However, the first results show an excellent signal to noise ratio on a significant subset of the events, demonstrating the general validity of the design and the electronics, which seems to be confirmed by a recent test in high intensity beam, similar to the conditions of the experiment where the GEM tracker will operate.

Work is in progress to finalize the design and to start the first production; to this end, specific effort is devoted to the optimization of the assembling procedure which includes the quality checks of the GEM and readout foils.

The GEM tracker project largely benefits of the expertise, experience and valuable facilities provided by the CERN RD51 collaboration [22].

\footnotetext{
${ }^{5}$ The cluster charge is the sum of the charge of the hits belonging to the cluster, baseline subtracted (step 5 above); the $\mathrm{x}$ strips are $80 \mu \mathrm{m}$ wide, the $\mathrm{y}$ strips are $340 \mu \mathrm{m}$ wide.
} 

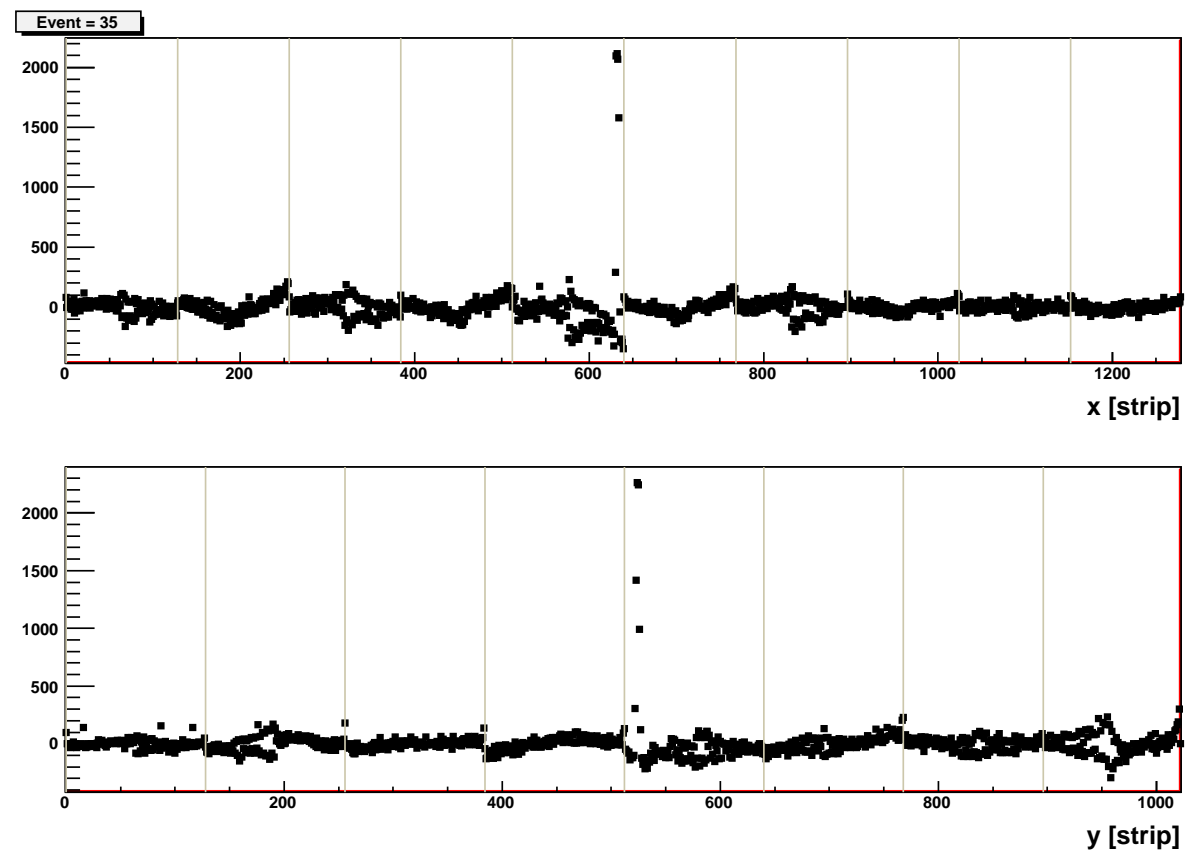

Good Event
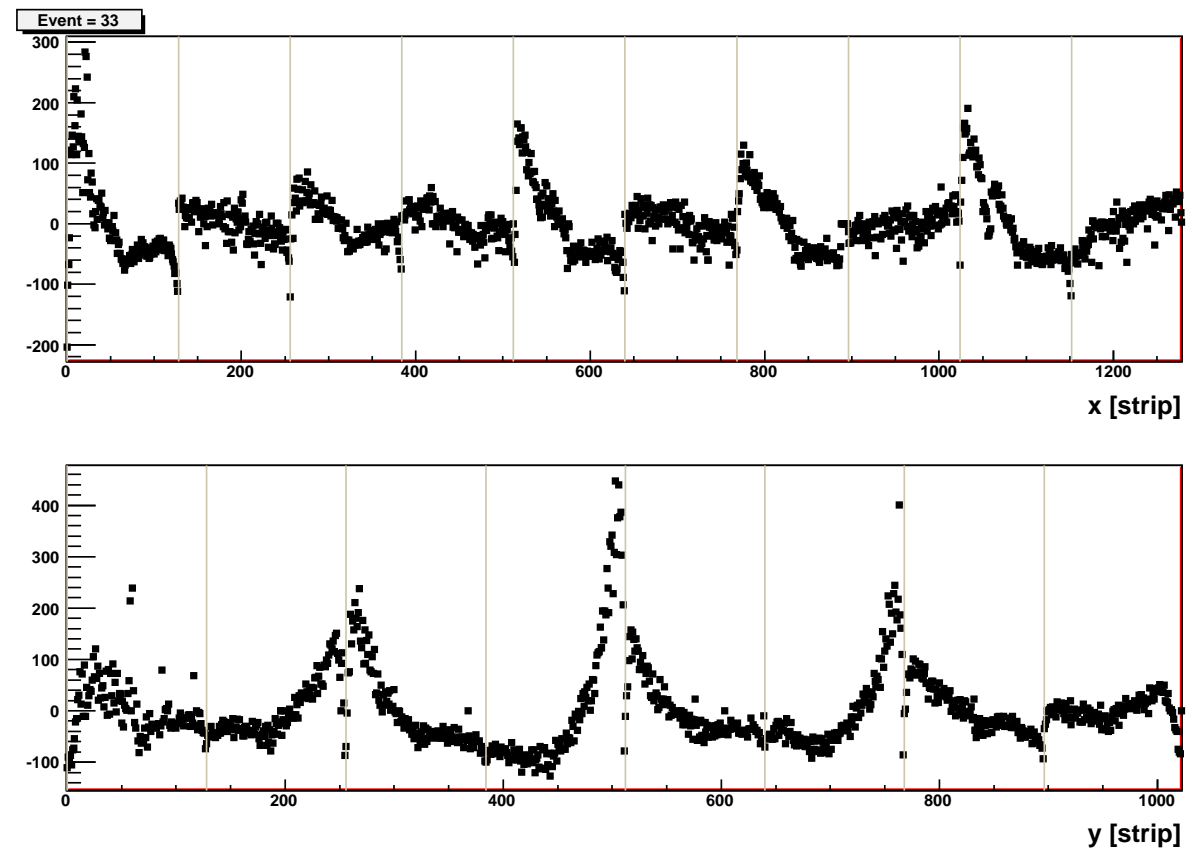

Noisy Event

Figure 9. Typical events recorded at the DESY test beam at the end of 2010. The upper 2 plots show a good event with very clean hit, the lower 2 plots represent a noisy event, likely due to uncompensated pedestal, as described in the text. The y-axis represents the collected charge in ADC unit. 


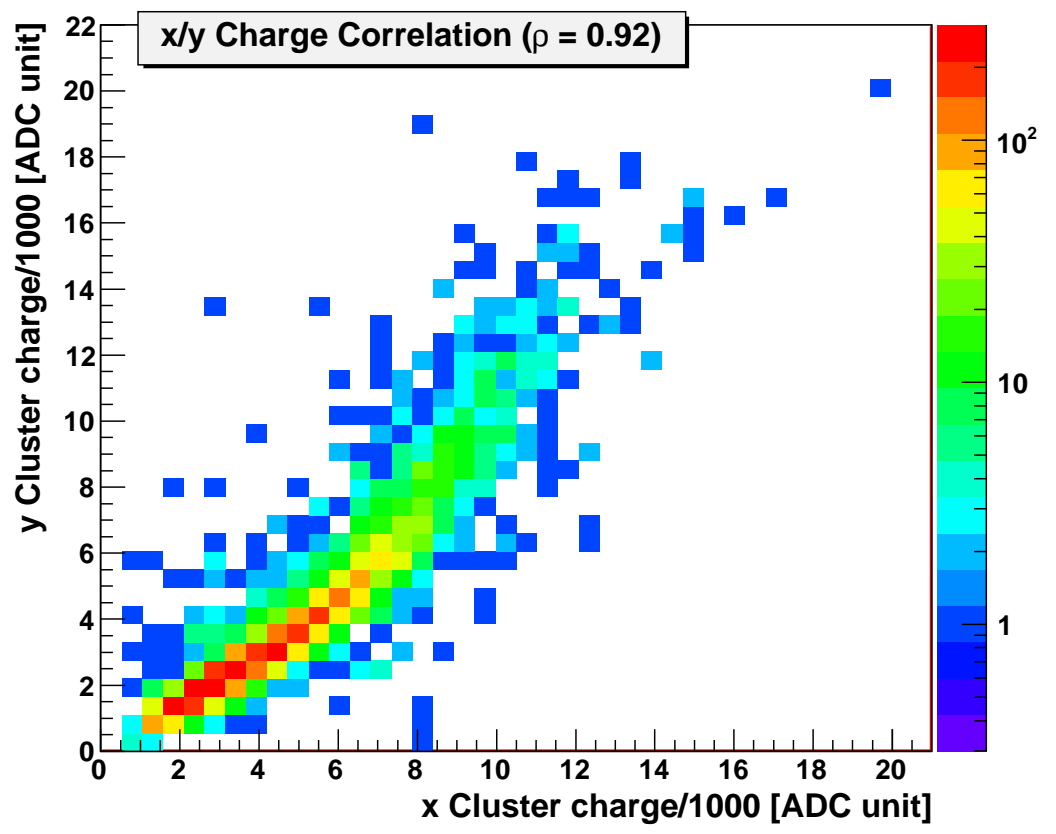

Figure 10. Preliminary plot of cluster charge correlation ( $\rho$ is the correlation coefficient).
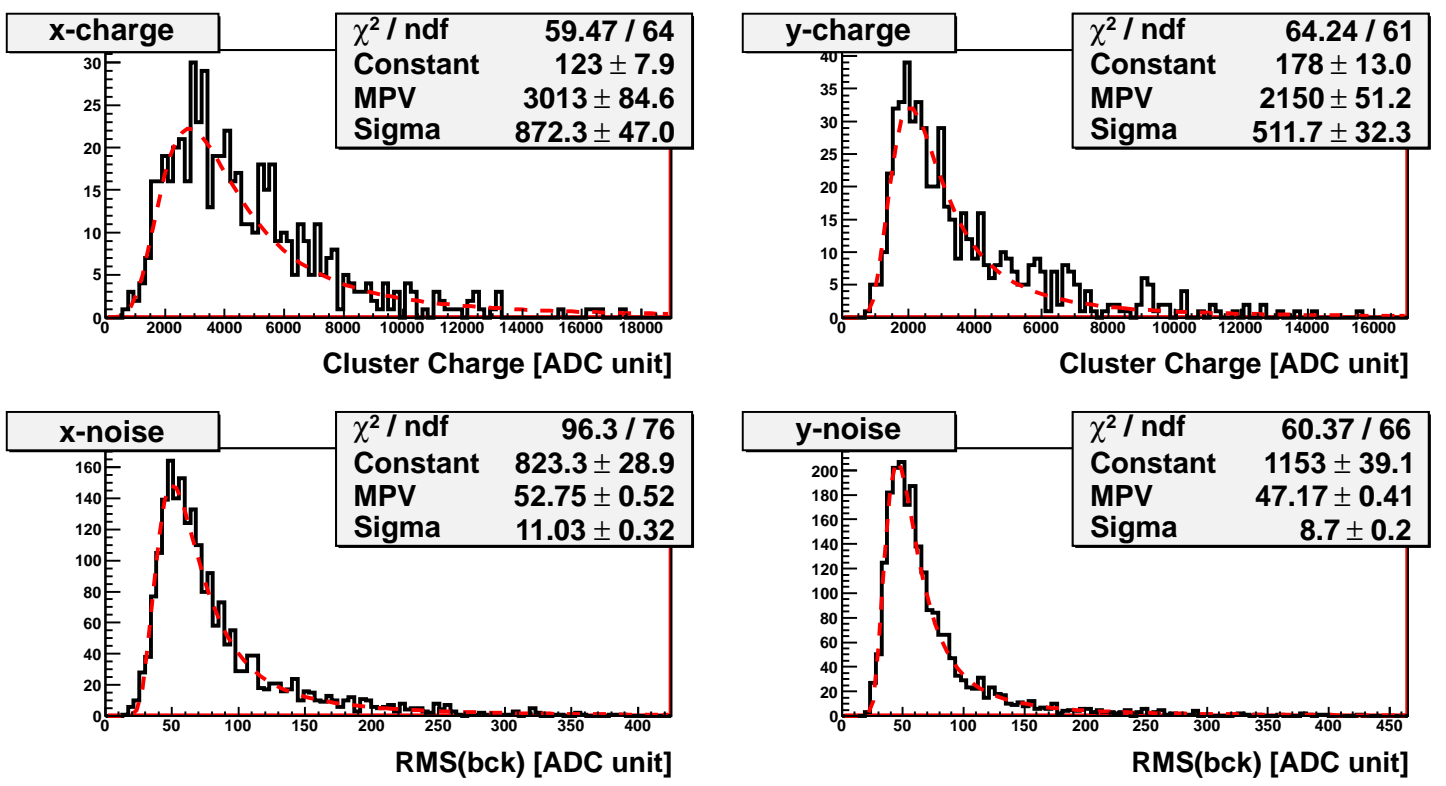

Figure 11. Preliminary cluster charge signal and noise distributions (solid lines), after quality cuts, as described in the text. Dashed lines represent the fit of Landau distributions. 


\section{References}

[1] Thomas Jefferson National Accelerator Facility web site: http://www.jlab.org.

[2] The SBS project web site: http://hallaweb.jlab.org/12GeV/SuperBigBite/.

[3] N. Akopov et al., The HERMES dual-radiator ring imaging Cherenkov detector, Nucl. Instrum. Meth. A 479 (2002) 511.

[4] $12 \mathrm{GeV}$ HallA upgrade web site: http://hallaweb.jlab.org/12GeV/.

[5] M.J. French et al., Design and results from the APV25, a deep sub-micron CMOS front-end chip for the CMS tracker, Nucl. Instrum. Meth. A 466 (2002) 359.

[6] A. Bressan et al., High rate behavior and discharge limits in micro-pattern detectors, Nucl. Instrum. Meth. A424 (1999) 321;

L. Poli-Lener et al., Triple-GEM detectors for the innermost region of the muon apparatus at the LHCb experiment, Ph.D. Thesis CERN-THESIS-2006-013 (2005).

[7] C. Altunbas et al., Construction, Test and Commissioning of the Triple-GEM Tracking Detector for COMPASS, CERN-EP/2002-008.

[8] Permali Composites, data sheet Durostone EPM 203 (also know as PERMAGLAS ME 730), http://www.permali.com/pdf/Durostone_sheets.pdf.

[9] JST web site: http://www.jst.com.

[10] Olympus experiment web site: http://web.mit.edu/OLYMPUS/.

[11] COMSOL web site: http://www.comsol.com.

[12] P. Spindel, Contribution to the set up of a Gas Electron Multiplier (GEM) detector, Master Thesis, University of Catania and Institut supérieur des Ingénieurs de Bruxelles, 2009; http://www.iss.infn.it/thesis/2009-master-spindel.pdf.

[13] V. De Smet, Study of a GEM tracker of charged particles for the Hall A high luminosity spectrometers at Jefferson Lab, MSc Thesis, University of Catania and Haute Ecole Paul Henri Spaak ISIB-Brussels (2011) http://www.iss.infn.it/thesis/2011-master-desmet.pdf.

[14] S. Agostinelli et al., GEANT4 - A simulation toolkit, Nucl. Instrum. Meth. A 506 (2003) 250.

[15] ROOT web site: http://root.cern.ch.

[16] A.H. Cookson and T.J. Lewis, Variations in the Townsend first ionization coefficient for gases, Brit. J. Appl. Phys. 17 (1966) 1473.

[17] F. Simon, Commissioning of the GEM Detectors in the COMPASS Experiment, Ph.D. Thesis Physik-Department E18, Technische Universität München (2001) http://www.e18.physik.tu-muenchen.de/publications/thesis/diploma_fsimon.pdf.

[18] V. Mamyan et al., GEM Detector Analysis, Talk presented at the 2011 summer Hall A collaboration meeting, web link: http://hallaweb.jlab.org/collab/meeting/2011-summer/talks/day2/GEM_Analysis.pdf.

[19] EUDET: Detector R\&D towards the Interntion Linear Collider, web site: http://www.eudet.org.

[20] G. Bencivenni, The GEM detector activity at the Frascati Laboratory, Nucl. Phys. A 827 (2009) 614C.

[21] G. Corradi et al., A novel High-Voltage System for a triple GEM detector, Nucl. Instrum. Meth. A 572 (2007) 96.

[22] RD51 collaboration web site: http://rd51-public.web.cern.ch/rd51-public/. 